\title{
A query theory account of the effect of memory retrieval on the sunk cost bias
}

\author{
Hsuchi Ting • Thomas S. Wallsten
}

Published online: 26 April 2011

(C) Psychonomic Society, Inc. 2011

The sunk cost bias is a phenomenon in which individuals continue to invest in an option when a better option is available (Arkes \& Blumer, 1985). One example is individuals' tendency to continue investing money in a stock that has been losing its value, rather than investing in a better alternative.

To many authors, it is not surprising that individuals allocate additional resources to an invested option, rather than explore alternatives, because people pay more attention to the sunk cost incurred as a result of their action than to the returns from the alternatives, due to loss aversion (Soman, 2004). However, simply assuming that sunk cost is interpreted as a loss and is, therefore, more important than potential gains does not explain the underlying processes. To remedy this, some researchers have suggested that this bias occurs because individuals interpret sunk cost as progress toward attaining the goal that motivated the original investment (Boehne \& Paese, 2000). Proponents of this hypothesis - the project completion hypothesishave shown that individuals become more willing to allocate resources to the invested option as goal attainment nears and goal completion becomes more important than economic concerns (Boehne \& Paese, 2000).

While the project completion hypothesis has stipulated that greater past investments increase individuals' belief that the goal has become more proximal, the theory does

\footnotetext{
H. Ting $(\bowtie)$

Tepper School of Business, Carnegie Mellon University, 5000 Forbes Avenue, Pittsburgh, PA 15213, USA

e-mail: hting@andrew.cmu.edu

T. S. Wallsten

Department of Psychology, University of Maryland, College Park, MD 20742, USA

e-mail: tswallst@umd.edu
}

not explain why economic concerns are rendered less important. Therefore, the aim of the present article is to provide an alternative account to explicate how past investments and individuals' perception of goal proximity due to past investments jointly influence individuals' tendency to display the sunk cost bias.

We used a model based on memory retrieval to explain why increasing past investments and proximity to the goal drive individuals to abandon economic rationality and display the sunk cost bias. This theory-query theory (Johnson, Häubl, \& Keinan, 2007) — is based on previous findings that the nature of a task influences how individuals evaluate their options (Fischer, Carmon, Ariely, \& Zauberman, 1999). For example, those who want to sell an object they own focus more on the object, whereas those who want to buy it focus more on the price they would need to pay. Objects' values are therefore influenced by the accessibility of the relevant information, and preference and choice are influenced by the most accessible information.

Query theory has three premises. First, one determines the value of an object by asking oneself questions, or queries, about it. In the context of sunk cost, one might ask, "Why should I allocate resources to the option in which I have already invested?" or "Why should I allocate resources to an alternative?" These queries are executed sequentially. In answering them, individuals will find reasons for pursuing either the option in which they have already invested or an alternative. Reasons for pursuing the invested option include either positive properties of the invested option or negative properties of an alternative. Reasons for pursuing the alternative include negative properties of the invested option or the positive properties of an alternative. Table 1 shows an example of each type of reason. 
Table 1 Examples of reasons provided by participants

\begin{tabular}{lll}
\hline Option & Properties of the Queries & Negative \\
\cline { 2 - 3 } & Positive & More calories than small chocolate \\
Invested & I can share it with someone else & Not enough for me \\
\hline Alternative & Easier to carry & \\
\hline
\end{tabular}

The top-left and bottom-right cells contain reasons for pursuing the invested option (big chocolate bar). The top-right and bottom-left cell contain reasons for pursuing the alternative (small chocolate bar)

Second, the sequential queries are generated serially and focus on the most important option or the current state of affairs first (Johnson et al., 2007; Weber \& Johnson, 2009). On the basis of previous research showing that since individuals consider options that have incurred sunk costs as the status quo (Whyte, 1986), they should produce queries about why they should pursue the invested options before considering the alternative, because it is the focal point in their decision-making. Thus, we hypothesize that reasons for pursuing the invested option will be generated before reasons for pursuing an alternative. In addition, building on the project completion hypothesis (Boehne \& Paese, 2000), this pattern should be more pronounced when individuals progress toward the goal that motivated their original investment as their motivation to attain it becomes stronger.

Third, the order in which the queries are generated affects valuations. Earlier queries are weighted more heavily in individuals' value construction than are later questions and, thus, exert a greater influence on valuation. Query theory therefore stipulates that reasons for pursuing the invested option should be more numerous and be retrieved earlier than reasons for pursuing any of the alternatives. The third premise is based on the memory retrieval process. Prior research has shown that a recalled item will increase recall of similar items by the process of shared context. However, recalled items can also decrease the likelihood that non retrieved items will be recalled later; and the greater the likelihood that an item will be recalled, the greater the inhibition of recall of the later non retrieved items (Anderson \& Spellman, 1995; Raaijmakers \& Shiffrin, 1981).

Query theory has been used to explain other decision biases. Previous research has shown that the endowment effect arises because ownership induces individuals to attend to the item they own and, subsequently, generate queries about that item first (Johnson et al., 2007). On inter temporal choice, researchers demonstrated that the time of consumption (i.e., now or some time later) influences the order and number of impatient thoughts about immediate consumption that are generated (Weber et al., 2007). On attribute framing, the authors found that political affiliations biased which tax scheme (carbon tax vs. carbon offset) is queried first, which, in turn, determines respondents' aversion toward tax or offset (Hardisty, Johnson, \& Weber, 2010). In sum, prior studies have shown that the option that draws the most attention will be queried first, thereby biasing individuals' perception about the choice. In all cases, forcing participants to query the option they paid less attention to reverses their preference.

We believe that the query theory can be applied to understanding and, therefore, attenuating individuals' tendency to display the bias, contrary to the loss aversion and project completion accounts, which offer no solution on how to mitigate the sunk cost bias. Experiment 1 shows that the balance of reasons supporting continued allocation of resources to an invested option versus to an alternative depends on the progress toward the goal and on sunk cost. Specifically, because individuals query the current state of affairs - in this case, the goal outcome some distance awaybefore querying alternative options, we hypothesize that the greater the progress toward the goal, the more the reasons will favor continuing, rather than pursuing the alternative. Also, the greater the progress at the time the reasons are generated, the earlier in the sequence of reasons will those for continuing appear. This imbalance in reason generation will, in turn, exaggerate the perceived value difference between the invested and an alternative option, thereby increasing individuals' tendency to display the sunk cost bias. Experiment 2 shows that forcing individuals to generate reasons in favor of an alternative option before an invested option reduces the sunk cost bias.

\section{Experiment 1}

Method

Participants Ninety-seven undergraduates from the University of Maryland participated in the study in exchange for course credit.

Design and procedure The participants were first shown a big (100-g) chocolate bar and a small (35-g) chocolate bar 
side by side. They learned that they could win the larger bar if they solved enough anagrams on the computer; otherwise, they would receive the smaller one. Therefore, the large chocolate bar was the invested option, while the small chocolate bar was the alternative option.

The participants were then randomly assigned to either approximate $(n=52)$ or a distant $(n=45)$ group; those in the proximate group were told that they needed to solve 20 anagrams to get a big chocolate bar, and those in the distant group were told that they needed to solve 30 . Unbeknownst to participants, the experiment had two parts. Participants first attempted Part 1 of the experiment, in which they were presented with 30 anagrams, of which half were easy and half difficult. All anagrams consisted of words of four to six letters drawn from the MRC Psycholinguistic Database, with familiarity ratings between 100 and 700 (Wilson, 1988). The difficult anagrams consisted of rare words with familiarity ratings between 100 and 200 (e.g., lagan, pavis), whereas the easy anagrams consisted of words with familiarity ratings between 600 and 700 (e.g., girl, beer). The anagrams were pretested on 45 participants, all of whom solved all the easy anagrams and none of whom solved more than $10 \%$ of the difficult anagrams. Therefore, participants in the proximate group were expected to fall 5 short of the number necessary for a big chocolate bar by the end of Part 1. By contrast, participants in the distant group were expected to fall 15 short of the number necessary for a big chocolate bar. The anagrams were presented sequentially, and participants could skip any one they wished by typing "skip."

At the end of Part 1, participants who had achieved their goal were given a large chocolate bar and were dismissed. The others began Part2. They were told the number of correct anagrams they had solved, the number they still needed to attain the big chocolate bar, and the amount of time they had spent on Part 1. This time measure indexed the total sunk cost for the invested option.

The participants then completed a brief questionnaire to measure positive and negative affect (PANAS; Watson, Clark, \& Tellegen, 1988). They next listed their thoughts, one at a time, on why they "would prefer either the big chocolate bar or the small chocolate bar." Participants could type between 1 and 10 reasons, which were displayed on the monitor as they were entered.

After giving reasons for why they wanted either the big or the small chocolate bar, participants then estimated the lengths of the two bars on the computer. Because objects are perceived to be larger when they become more valuable (Bruner \& Goodman, 1947), the estimations of length served as an index for the values that the participants attributed to the invested and alternative options.

Finally, participants were informed that they could choose to complete Part 2 of the experiment, which provided 25 additional anagrams from which they might solve enough to earn the big chocolate bar. Two unsolvable anagrams appeared sometime during the first four anagrams. The time that the participants spent on these unsolvable anagrams constituted our index of sunk cost bias. The experiment ended when participants solved a sufficient number of anagrams to win the big chocolate bar, exhausted all anagrams, or gave up.

\section{Results}

Eight participants in the proximate group solved the necessary number of anagrams during Part 1 of the experiment and, therefore, did not contribute any data. The PANAS scores did not differ between the proximate and distant conditions, nor did they have any significant correlation with other measured variables. We did not analyze the PANAS further.

Order of reasons We hypothesized that individuals should generate reasons that supported the invested option before generating reasons for pursuing any alternative. The first author and a coder blind to our hypotheses first coded the reasons entered by the participants as either reasons supporting the invested option or reasons for pursuing any alternative $(\alpha=.91)$. All subsequent analyses were based on the blind coder's coding.

We then used the standardized median rank difference (SMRD) to calculate the order of reasons. The SMRD is defined as $2\left(M R_{\mathrm{a}}-M R_{\mathrm{i}}\right) / n$, where $M R_{\mathrm{i}}$ is the median rank of reasons for pursuing the invested option, $M R_{\mathrm{a}}$ is the median rank of reasons for pursuing the alternative, and $n$ is the total number of reasons generated. SMRD scores range from +1 to -1 . Positive scores indicate that, on average, reasons for pursuing the invested option are listed prior to reasons for pursuing the alternative. Negative scores indicate the opposite.

Since we hypothesized that SMRD should be higher in the proximate than in the distant condition, we used a onetailed test to analyze the results. The bar graph in Fig. 1a reveals that the average SMRD score was higher for participants in the proximate group than for those in the distant group (.69 vs. .43, respectively), $t(87)=1.67$, $p=.049$, one-tailed, $d=0.36$. This pattern supports our hypothesis that queries are executed serially, with initial queries focused on invested options.

Types of reasons Participants in the proximate group, whose order of reasons had a higher SMRD score, should give more reasons for pursuing the invested option and give fewer reasons for pursuing the alternative than should participants in the distant group. Figure $1 \mathrm{~b}$ shows that the 
Fig. 1 Results of Experiment 1 All error bars indicate $\pm 1 S E$. a Standardized median rank difference (SMRD) as a function of distance from the goal. $\mathbf{b}$ Number of reasons that support pursuing the invested and alternative options as a function of distance from the goal. c Difference in estimated length between the big and small chocolate bars as a function of distance from the goal. d Amount of time spent on unsolvable anagrams

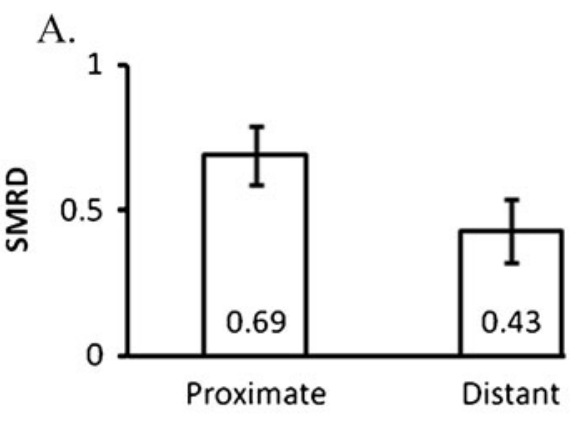

B.

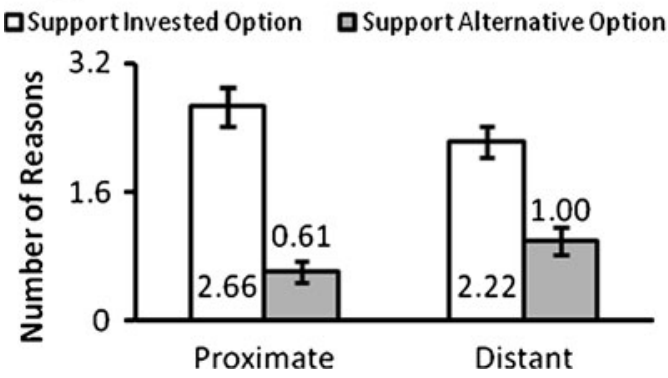

C.

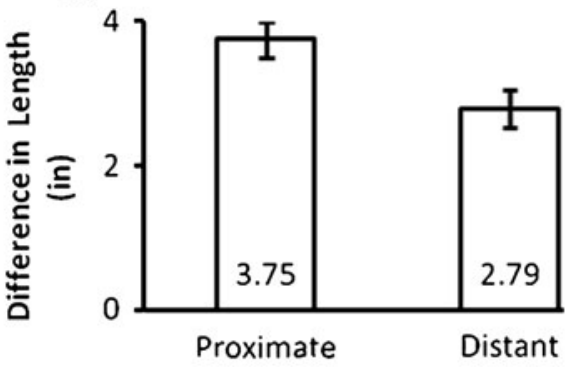

D.

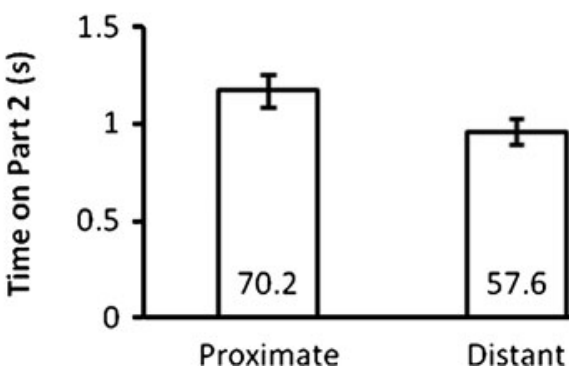

participants in the proximate group generated more reasons for pursuing the invested option (2.66 vs. 2.22 , respectively) and fewer reasons for pursuing the alternative (.61 vs. 1.00 , respectively) than did participants in the distant group. A 2 (distance: proximate vs. distant) $\times 2$ (support: for invested vs. for alternative) mixed ANOVA with the repeated measure on the second factor shows a significant interaction, $F(1,87)=4.00, p=.049, d=0.33$.

Difference in length More reasons generated to support the pursuit of the invested option and fewer reasons generated to support the pursuit of the alternative should, in turn, influence the perceived value difference between the two options. This difference should be more pronounced in the proximate condition than in the distant condition. Recall that the size estimate indexed the judged value of the option. Fig. 1c shows that the difference between the estimated length for the big and small chocolate bars was larger for participants in the proximate group than for those in the distant group ( 3.75 vs. 2.80 in., respectively), $t(86)=$ $2.75, p=.004, d=0.59 .{ }^{1}$ The proximity to the reward yielded by the invested option widened the gap in the subjective assessment of the value of the two options.

Time spent The amount of time that participants spent on Part 1 of the experiment predicted how much time they spent on the

\footnotetext{
${ }^{1}$ One participant in Experiment 1 and another participant in Experiment 2 did not estimate the lengths of the big and small chocolate bars. They were excluded from the analysis of length estimation but were included in the other analyses.
}

unsolvable anagrams in Part $2(\beta=.21, t=2.02, p=.047)$. However, an analysis of covariance showed that the prediction breaks down when the distance from the goal is included in the regression, consistent with the project completion hypothesis that the sunk cost plays no role in the sunk cost bias after accounting for the distance from the goal.

Figure 1d shows that the proximate group spent more time on the unsolvable anagrams in Part 2 of the experiment, as compared with the distant group (70.2 vs. $57.6 \mathrm{~s}$, respectively), $t(87)=2.07, p=.04, d=0.44$.

\section{Predicting time spent on Part 2 from the length difference} Regression analysis showed that the difference in estimated length significantly predicted the time that the participants spent on the unsolvable anagrams, $\beta=.22, t=2.08, p=.04$.

\section{Discussion}

Experiment 1 provides evidence that memory retrieval may underlie individuals' inclination to invest in one option at the expense of alternatives. In addition, the sunk cost individuals invested in terms of time spent in Part 1 of the experiment relates positively to the amount of time they spent on Part 2. However this relationship disappears when the distance from attaining the goal yielded by the invested option is controlled for. Moreover, distance from the goal influences the order in which reasons are generated and the types of reason that are considered by individuals, which, in turn, influence the perceived difference in value between 
the two options. The regression analysis showed that this difference significantly predicts the tendency to display the sunk cost bias.

Results thus far seem to corroborate our hypothesis that individuals' progress and accumulated sunk costs toward attaining an invested option influences the queries they generate to evaluate the invested and alternative options. The reasons generated in answering the queries, in turn, affect how the two options are valued and the individuals' propensity to display the sunk cost bias. However, one can argue that participants experienced progress toward only the invested, but not the alternative, option; thus, the exaggerated difference in value estimations might be due entirely to participants' inability to invest time toward attaining an alternative option. In addition, if the order of reasons generated by individuals drives the perception of value and sunk cost bias, forcing individuals to generate reasons for pursuing the alternative option first should attenuate the difference in the estimated length between the invested and alternative options and should reduce the time participants spend on unsolvable anagrams and the propensity to stay with the invested option. Experiment 2 tested both notions.

\section{Experiment 2}

\section{Participants}

One hundred-forty-three undergraduates from the University of Maryland participated in this study in exchange for course credit.

\section{Design and procedure}

The experiment was a 2 (distance: proximate vs. distant) $\times 2$ (reasons generation: invested option first vs. alternative option first) ANOVA design. Participants first were told that they needed to solve 40 anagrams correctly in order to receive the big chocolate bar or fewer for the small bar and were randomly assigned to either the proximate $(n=70)$ or the distant $(n=73)$ condition. All participants then proceeded to Part 1 of the experiment, in which those in the proximate (distant) condition had to successfully solve 30 (15) anagrams. Upon completing the requisite number of anagrams, participants learned the amount of time they spent on Part 1and the amount of anagrams still needed for the big chocolate bar. Then half of the participants were assigned to the invested-option-first condition and were instructed to generate reasons why they preferred the big chocolate bar before they generated reasons why they preferred the small chocolate bar. The remaining half was assigned to the alternative-option-first condition and were instructed to generate reasons in the reverse order.
All the participants at this point estimated the lengths of the two bars on the computer and then decided whether to solve the remaining number of anagrams for the big chocolate bar or to solve just 5 anagrams for the small chocolate bar. Once the choice was made, participants began Part 2 of the experiment, which provided additional anagrams from which they might solve a sufficient number of anagrams, 10 for the proximate and 25 for the distant group, to earn the big chocolate bar. Two unsolvable anagrams appeared sometime during the first 4 anagrams. Part 2 ended when participants either solved a sufficient number of anagrams for their chosen chocolate bar or gave up.

\section{Results}

Types of reasons As in Experiment 1, we first coded reasons containing positive properties of the invested (alternative) option and negative properties of an alternative (invested) option as reasons for pursuing the invested (alternative) option. The first author and a coder blind to the research hypotheses coded the reasons $(\alpha=.93)$. All subsequent analyses were based on the blind coder's coding.

Figure 2a shows the difference in the numbers of reasons given in favor of pursuing the invested versus the alternative option. A 2 (distance: proximate vs. distant) $\times 2$ (query order: invested option first vs. alternative option first) ANOVA with difference between the number of reasons for pursuing the invested option and the number of reasons for pursuing the alternative as the dependent variable reveals that query order was significant, $F(1,139)=$ $21.83, p<.001, d=0.77$. Participants in the invested-optionfirst condition generated more reasons supporting the invested option than supporting the alternative option; the pattern was reversed in the alternative-first condition (.86 vs. -.56 , respectively).

Difference in length Controlling the order of query generation affected the perceived length difference (a proxy for value difference) in the expected direction. That is, the difference was less in the alternative-option-first than in the invested-option-first condition, as indicated by the significant query order, $F(1,138)=5.02, p=.027, d=0.37$. Figure $2 \mathrm{~b}$ shows the estimated line lengths under the two conditions.

Time spent and switching As in Experiment 1, sunk cost in terms of the amount of time spent on Part 1 significantly predicted the time that participants spent on unsolvable anagrams in Part 2 and the likelihood that they would choose the invested over the alternative option. However, an ANCOVA showed that these relationships became non significant when goal distance was added as a covariate. 
Fig. 2 Results of Experiment 2. All error bars indicate $\pm 1 S E$. a Effects of distance from the goal and query order on the difference between the numbers of reasons generated to support pursuing the invested and alternative options. b Effects of distance from the goal and query order on the difference in estimated length between the big and small chocolate bars. $\mathbf{c}$ Effects of distance from the goal and query order on the amount of time spent on unsolvable anagrams. d Effects of distance from the goal and query order on the proportion of participants who chose to pursue the invested option over the alternative option upon finishing Part 1 of the experiment

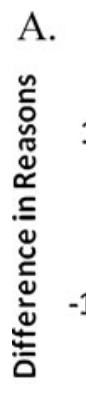

A.
口Proximate QDistant

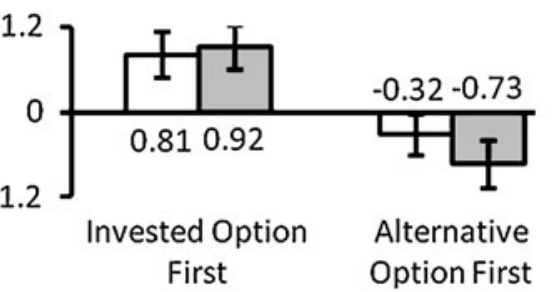

B.
口Proximate QDistant

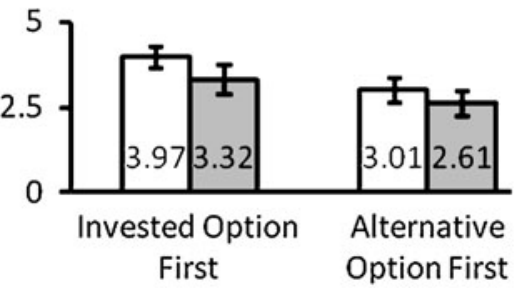

C.

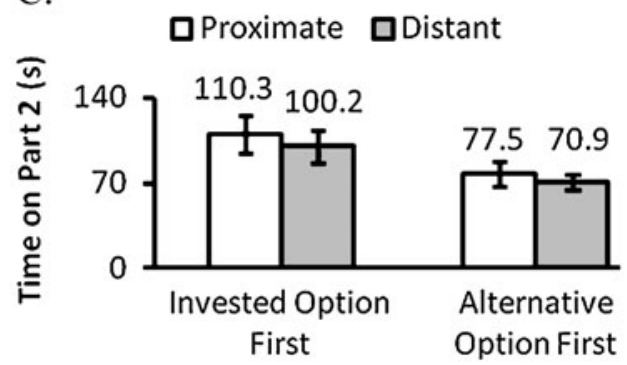

D.

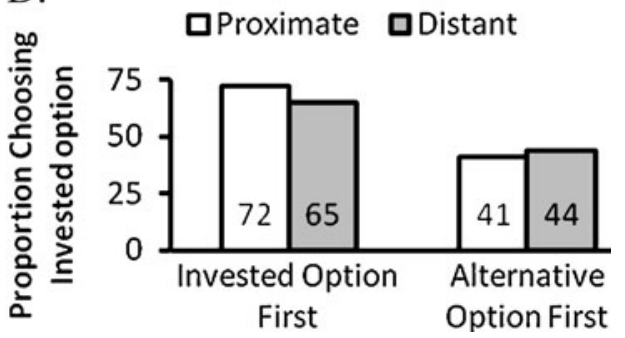

Figure 2c shows that participants who generated queries on the invested option first spent significantly more time on the unsolvable anagrams than did those who generated queries on the alternative option first, as evidenced by the significant query order, $F(1,139)=6.66, p=.01, d=0.41$.

Figure $2 \mathrm{~d}$ shows the choice behavior. Consistent with the previous result, logistic regression revealed that only query order was significant, $B=.54, S E=.18, p=.002$. A greater proportion of participants in the invested-option-first condition chose the invested option than did those in the alternative-option-first condition.

Predicting time spent and choice from the difference in perceived length Regression analysis showed that the difference in estimated length significantly predicted the time that participants spent on the unsolvable anagrams, $\beta=.195, t=2.35, p=.02$. A separate logistic regression also showed that length difference significantly predicted the likelihood to choose pursuing the invested option, $B=.16 . S E=.08, p=.047$.

PANAS scores The PANAS scores did not significantly differ between the proximate and distant conditions and did not correlate with any measured variables.

\section{Discussion}

The results corroborate our hypothesis that the type and order of reasons generated to support an option influences the perceived difference in values between the two options, which, in turn, determines individuals' tendency to display the sunk cost bias. The difference in perceived length of the large and small chocolate bars was reduced when the participants were prompted to generate reasons that supported the small bar first, consequently reducing individuals' tendency to display the sunk cost bias, as observed in Experiment 1.

\section{General discussion}

Research on the sunk cost bias has focused mainly on why individuals continue allocating resources to the invested option, and few studies have investigated why they ignore potential opportunities to invest in alternatives. In two experiments, we showed that people generate reasons for pursuing an invested option more frequently and earlier than they do for an alternative in a sequence of reasons and that these differences increase as individuals progress toward the goal yielded by the invested option. This process of retrieving reasons leads to an exaggerated perception of the value difference between the invested and alternative options and causes individuals to display the sunk cost bias - in this case, continuing to spend time on unsolvable anagrams in the hope of attaining the large chocolate bar. However, reversing the natural order of generating reasons for pursuing the alternative option reduced the subjective difference in value and resulted in a reduced tendency to display the bias.

Past research has shown that asking individuals to carefully consider the "pros and cons" of all available options after incurring sunk costs does not reduce the bias (e.g., Simonson \& Staw, 1992). Our results are consistent with this pattern, since they show that individuals rarely consider positive features of an alternative once they have sunk resources into an invested option. Even when positive features of the alternative are considered, they are contemplated at a much 
later time, as compared with the positive features of the invested option.

The present findings also relate to research on opportunity cost. Opportunity cost is the (missed) benefits of the foregone choice. Most individuals have difficulty accounting for opportunity costs because they overvalue costs that arise from their own actions, a form of loss aversion (Thaler, 1994). In our view, the neglect of opportunity cost emerges because individuals generate more positive reasons for the option they own or have invested in than for alternatives, due to output interference in memory. This effect, in turn, causes individuals to perceive the benefits lost from the foregone option to be less than the potential benefits gained from the option they have chosen. In short, opportunity costs are often ignored not because individuals do not attend to them but, rather, because they judge foregone options to be less valuable than options they currently own or are pursuing, due to the types of queries and answers they generate.

According to Arkes and Blumer (1985), sunk costs can come in many forms, from money to time to effort. Therefore, we hasten to add that while manipulating the query orders can be a useful debiasing mechanism in counteracting the sunk cost bias when the past investment is one of time, additional research is necessary to determine whether the effect holds for other forms of sunk costs. In addition, one might wonder if our results would have been the same if we had asked participants to list queries only as a "thought experiment," without writing them down. Prior research has shown that the cognitive strategy of "consider the opposite" can reduce overconfidence and anchoring effects (Larrick, 2004), which suggests that decision makers can counteract the sunk cost bias by forcing themselves to mentally list queries on alternative options first.

Sunk cost bias has garnered a tremendous amount of interest in economics, political science, and psychology. Over the past 30 years, numerous studies have shown that most individuals are overly attentive to the option in which they have sunk costs and are reluctant to adopt alternative options. We depart from previous efforts by explaining the sunk cost bias in terms of underlying memory retrieval processes and value judgments. Understanding the processes behind the sunk cost bias is an important endeavor, because common strategies that have been proposed to reduce it, such as presenting alternatives (McCain, 1986), encouraging rational thinking (Brockner, Shaw, \& Rubin, 1979), and setting minimum target progress before investing further (Boulding, Morgan, \& Staelin, 1997), are only minimally effective (Boulding et al., 1997; Simonson \& Staw, 1992). As the field continues to accumulate evidence of the bias, we hope that highlighting the cognitive process will offer fresh considerations and strategies for avoiding it.
Author Note We wish to thank Melanie Alberts, Josh Boker, Michelle Bollen, and Jacki LaRusso for assistance in data collection.

\section{References}

Anderson, M. C., \& Spellman, B. A. (1995). On the status of inhibitory mechanisms in cognition: Memory retrieval as a model case. Psychological Review, 102, 68-100.

Arkes, H. R., \& Blumer, C. (1985). The psychology of sunk cost. Organizational Behavior and Human Decision Processes, 35, 124-140.

Boehne, D. M., \& Paese, P. W. (2000). Deciding whether to complete or terminate an unfinished project: A strong test of the project completion hypothesis. Organizational Behavior and Human Decision Processes, 81, 178-194.

Boulding, W., Morgan, R., \& Staelin, R. (1997). Pulling the plug to stop the new product drain. Journal of Marketing Research, 34, 164-176.

Brockner, J., Shaw, M. C., \& Rubin, J. Z. (1979). Factors affecting withdrawal from an escalating conflict: Quitting before it's too late. Journal of Experimental Social Psychology, 15, 492-503.

Bruner, J., \& Goodman, C. C. (1947). Value and need as organizing factors in perception. Journal of Abnormal and Social Psychology, $42,33-44$

Fischer, G. W., Carmon, Z., Ariely, D., \& Zauberman, G. (1999). Goal-based construction of preferences: Task goals and the prominence effect. Management Science, 45, 1057-1075.

Hardisty, D. J., Johnson, E. J., \& Weber, E. U. (2010). A dirty word or a dirty world? Attribute framing, political affiliation, and query theory. Psychological Science, 21, 86-92.

Johnson, E. J., Häubl, G., \& Keinan, A. (2007). Aspects of endowment: A query theory of value construction. Journal of Experimental Psychology. Learning, Memory, and Cognition, 33, 461-474.

Larrick, R. P. (2004). Debiasing. In D. J. Koehler \& N. Harvey (Eds.), Blackwell handbook of judgmentand decision making (pp. 316337). Malden: Blackwell.

McCain, B. E. (1986). Continuing investment under conditions of failure. Journal of Applied Psychology, 71, 280-284.

Raaijmakers, J. W., \& Shiffrin, R. M. (1981). Search of associative memory. Psychological Review, 88, 93-134.

Simonson, I., \& Staw, B. M. (1992). Deescalation strategies: A comparison of techniques for reducing commitment to losing courses of action. Journal of Applied Psychology, 77, 419-426.

Soman, D. (2004). Framing, loss aversion, and mental accounting. In N. Harvey \& D. Koehler (Eds.), Blackwell handbook of judgment and decision making research (pp. 379-398). London: Blackwell.

Thaler, R. (1994). The psychology of choice and the assumptions of economics. In R. Thaler (Ed.), Quasi rational economics (pp. 137-166). New York: Russell Sage Foundation.

Watson, D., Clark, L. A., \& Tellegen, A. (1988). Development and validation of brief measures of positive and negative affect: The PANAS scales. Journal of Personality and Social Psychology, 54, 1063-1070.

Weber, E. U., \& Johnson, E. J. (2009). Mindful judgment and decision making. Annual Review of Psychology, 60, 53-86.

Weber, E. U., Johnson, E. J., Milch, K. F., Chang, H., Brodscholl, J. C., \& Goldstein, D. G. (2007). Asymmetric discounting in inter temporal choice. Psychological Science, 18, 516-523.

Whyte, G. (1986). Escalating commitment to course of action: A reinterpretation. Academy of Management Review, 11, 311321.

Wilson, M. D. (1988). The MRC psycholinguistic database: Machine readable dictionary, version 2. Behavioral Research Methods, Instruments, \& Computers, 20, 6-11. 